\title{
Comparison of concentrations of two proteinase inhibitors, porcine pancreatic elastase inhibitory capacity, and cell profiles in sequential bronchoalveolar lavage samples
}

\author{
HM MORRISON, JA KRAMPS, JH DIJKMAN, RA STOCKLEY
}

From the General Hospital, Birmingham, and Department of Pulmonary Diseases, University Hospital, Leiden, The Netherlands

ABSTRACT Bronchoalveolar lavage is used to obtain cells and proteins from the lower respiratory tract for diagnosis and research. Uncertainty exists about which site in the lung is sampled by the lavage fluid and what effect different lavage volumes have on recovery of the constituents of lavage fluid. Dilution of alveolar lining fluid by lavage fluid is variable and results are usually expressed as protein ratios to surmount this problem. We have compared cell profiles and the concentrations of two proteinase inhibitors - the low molecular weight bronchial protease inhibitor antileucoprotease and $\alpha_{1}$ proteinase inhibitor, together with $\alpha_{1}$ proteinase inhibitor function and its relationship to the cell profile in sequential bronchoalveolar lavage fluid samples from patients undergoing bronchoscopy. There was no difference in total or differential cell counts or albumin or $\alpha_{1}$ proteinase inhibitor concentrations between the first and second halves of the lavage. Both the concentration of antileucoprotease and the ratio of antileucoprotease to albumin were, however, lower in the second half of the lavage $(2 p<0.01$ and $2 p<0.05$ respectively). There was no difference in the function of $\alpha_{1}$ proteinase inhibitor (assessed by inhibition of porcine pancreatic elastase-PPE) between aliquots $\left(0.28 \mathrm{~mole}\right.$ PPE inhibited $/ \mathrm{mol} \alpha_{1}$ proteinase inhibitor; range $0-1.19$ for the first half and $0.37 \mathrm{~mol}$ PPE inhibited $/ \mathrm{mol} \alpha_{1}$ proteinase inhibitor; range $0.10-0.80$ for the second half). About $60-70 \%$ of $\alpha_{1}$ proteinase inhibitor in each half of the lavage fluid was inactive as an inhibitor. The function of $\alpha_{1}$ proteinase inhibitor did not differ between bronchitic smokers and ex-smokers. Alpha $_{1}$ proteinase inhibitor function was not related to the number of total white cells, macrophages, or neutrophils in the lavage fluid. Contamination of lavage by red blood cells was found to alter the concentration of $\alpha_{1}$ proteinase inhibitor but not its function when aliquots with and without erythrocytes were compared. These results show that the only difference between the two halves of these lavage samples is in the amount of antileucoprotease present, suggesting that more proximal secretions are being harvested early in the lavage procedure. Much of the $\alpha_{1}$ proteinase inhibitor present in the samples is functionally inactive, but this is not clearly related to any particular cell type or to smoking habits, and does not differ between different stages of the lavage procedure. Finally, the presence of erythrocytes probably does affect $\alpha_{1}$ proteinase inhibitor concentration and such samples should be excluded from analysis.

Investigation of proteolytic enzymes and antiproteases in lung secretions has provided valuable insight into the pathogenesis of such diverse diseases

\footnotetext{
Address for reprint requests: Dr RA Stockley, General Hospital, Birmingham B4 6NH.
}

Accepted 29 November 1985 as emphysema, cryptogenic fibrosing alveolitis, sarcoidosis, and adult respiratory distress syndrome. ${ }^{12}$ The use of bronchoalveolar lavage has enabled proteins and cells to be sampled directly from the bronchoalveolar region of the lung and this overcomes the problem of contamination by saliva and nasopharyngeal secretions that may affect results in 
sputum. Much has been learned, but unfortunately various problems with both the technique itself and the expression of the results obtained means that caution must be exercised in interpretation of the information generated, comparing results obtained by different research groups, and considering the place of the technique as a diagnostic and research tool.

Several factors may influence the results. Firstly, the method of bronchoscopy and bronchoalveolar lavage differs between laboratories and individual endoscopists, producing results that are not comparable.

Secondly, the amount of lavage fluid returned varies greatly. Local anaesthetic and the lavage fluid itself cause variable dilution of the fluid lining the bronchi and alveoli. Protein measurements are usually expressed as ratios to surmount the uncertainty introduced by dilutional factors, but other methods have been used in an attempt to standardise measurements. A particular protein may be related to the concentration of total protein, albumin, monomeric immunoglobulin A (IgA), secretory IgA, or potassium ion. ${ }^{3}$ Some workers have also added methylene blue to the fluid used for lavage and measured its dilution in the fluid recovered, to quantify the volume of secretion returned. ${ }^{4}$ Standardising against the albumin concentration in the lavage fluid, however, is the technique used most frequently.

Thirdly, there is uncertainty about the area of the lung being sampled. Since the bronchoscope cannot usually be passed beyond the fourth generation bronchi, clearly many orders of bronchi and peripheral bronchi are lavaged during the procedure, although some of the fluid instilled is assumed to reach the alveoli and to be retrieved. In addition, it has been suggested that peripheral bronchi are sampled during the initial part of a lavage and alveolar cells and proteins are recovered from the subsequent fluid instilled. ${ }^{5}$ Since neutrophils may predominate in the more proximal airways and macrophages are the major cell type in the alveoli, ${ }^{1}$ the release of proteolytic enzymes and oxidants from the different cell populations and their interaction with proteinase inhibitors could lead to variation in results of functional inhibitor studies between the early and late fractions of the lavage fluid.

Finally, contamination of secretions obtained by lavage may occur if the bronchial mucosa is traumatised during the procedure. The presence of red blood cells in the lavage fluid is often thought to indicate that serum proteins have entered the bronchial tree as a result of mucosal damage ${ }^{1}$ and such samples are frequently discarded, although it is not clear whether this is necessary.

The present study had two objectives. The first was to clarify whether separate sites of the bronchial tree are sampled by the early and late phases of the lavage $\overrightarrow{\vec{S}}$ procedure. The concentrations of $\alpha_{1}$ proteinase $\frac{\vec{O}}{0}$ inhibitor and low molecular weight bronchial pro- $\frac{}{0}$ tease inhibitor, and also albumin concentration, were $\frac{\overline{\bar{p}}}{\bar{p}}$ compared in sequential aliquots of lavage fluid from $\mathbb{\otimes}$ patients undergoing diagnostic fibreoptic bronchoscopy. Secondly, we wished to determine whether the presence of either red blood cells (suggestive of $\bar{P}$ trauma with consequent leakage of serum) or of white $\overrightarrow{\vec{\omega}}$ blood cells (which produce oxidants and enzymes $\stackrel{\sigma}{\omega}$ capable of inactivating $\alpha_{1}$ proteinase inhibitor) ${ }^{67}$ was $\vec{x}$ related to $\alpha_{1}$ proteinase inhibitor function.

\section{Methods}

We studied a total of 19 patients (mean age 61.8 (SD 10.8 ) years); eight were women. All were undergoing $c$

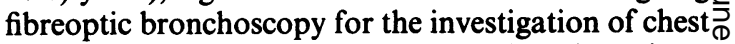
symptoms or radiographic abnormalities. Six patients $\vec{\bullet}$ had evidence of interstitial lung disease and showed aog restrictive defect on pulmonary function testing with. interstitial changes on the chest radiograph. Four of them had cryptogenic fibrosing alveolitis and two had sarcoidosis. Two patients presented with haemoptysiso for which no cause was found, and the remaining $11 \frac{\circ}{\circ}$ patients had carcinoma of the bronchus. The latter $\cong$ two groups had similar lung function (meano함 $\mathrm{FEV}_{1} / \mathrm{FVC}$ ratio $69.6 \%(17.3 \%)$ ) and they are 3 considered together.

Fibreoptic bronchoscopy and bronchoalveolar lavage were performed by the same person (RAS). The patients received intramuscular atropine $(600 \mu \mathrm{g})$ 응 and intravenous diazepam $(5-10 \mathrm{mg})$ as pre- $x$ medication and the bronchoscope was passed into the lower respiratory tract with the aid of lignocaine as an. local anaesthetic. It was wedged in the middle lobe or lingula (and, in the case of the patients with tumours, in the radiologically or macroscopically normal lung) and three $20 \mathrm{ml}$ boluses of warmed sterile physio logical saline were instilled and gently aspirated in turn. The volume returned formed the first aliquot of lavage fluid. The procedure was repeated to obtain the second aliquot.

Each aliquot was then processed in a similar way $\frac{\omega}{\sigma}$ The lavage fluid was filtered through a double layer of gauze to remove mucus and the volume of fluid wast noted. One millilitre was removed for a total celes count, a Coulter counter being used. The remaining fluid was centrifuged at $3000 \mathrm{rev} / \mathrm{min}$ for five minutes and the cell pellet was resuspended in a drop of the supernatant. The cell suspension was placed on an? albuminised slide, fixed in $95 \%$ alcohol and stained? with Papanicolau's stain. One slide was air dried and stained with Giemsa. At least 200 cells were therb counted to obtain a differential cell count. The lavage 
fluid remaining after removal of the cells was concentrated in an Amicon pressure filtration system, with a YCO5 membrane (molecular weight cut off 500 daltons). The concentration factor was recorded.

The concentrations of $\alpha_{1}$ proteinase inhibitor and albumin in the lavage fluid were determined by rocket immunoelectrophoresis. The antibody used to quantitate $\alpha_{1}$ proteinase inhibitor was known to give accurate results even for protein that had recently interacted with enzyme. ${ }^{8}$ The ratio of $\alpha_{1}$ proteinase inhibitor to albumin was calculated for each sample. A portion of each aliquot of lavage fluid was coded, frozen in dry ice, and flown to Leiden for the measurement of the low molecular weight inhibitor (antileucoprotease) by an enzyme-linked immunosorbent assay. ${ }^{9}$ The ratios of antileucoprotease to albumin were also calculated for each pair of aliquots.

The method of determining $\alpha_{1}$ proteinase inhibitor function has been described. ${ }^{10}$ In summary, increasing amounts of lavage fluid were preincubated with a fixed amount of porcine pancreatic elastase of known activity ${ }^{10}$ obtained from PL Biochemicals, Milwaukee, Wisconsin. After further incubation with the substrate succinyl-trialanyl-paranitroanilide the residual enzyme activity was determined spectrophotometrically and expressed as a percentage of the activity of porcine pancreatic elastase and substrate alone. The amount of lavage fluid necessary to inhibit the enzyme totally (that is, to give zero absorbance) was determined by interpolation by the method of least squares and expressed as micrograms of porcine pancreatic elastase inhibited per millilitre of lavage fluid, after correction for concentration (between batch coefficient of variation $=5.6 \%$ ).

The amount of active $\alpha_{1}$ proteinase inhibitor in $1 \mathrm{ml}$ of unconcentrated lavage fluid was determined by calculating the number of moles of porcine pancreatic elastase inhibited by $1 \mathrm{ml}$ concentrated lavage fluid, on the assumption of a 1:1 molar interaction between porcine pancreatic elastase and $\alpha_{1}$ proteinase inhibitor, ${ }^{11}$ and corrected with the concentration factor. This was compared to the total amount of $\alpha_{1}$ proteinase inhibitor in the unconcentrated lavage fluid measured immunologically, and the amount of inactive $\alpha_{1}$ proteinase inhibitor in each sample equalled the difference between the two values.

Wilcoxon's rank sum test for paired samples (two tailed) was used to assess any difference between the two aliquots, and least squares analysis (two tailed) was used to determine the relationship between $\alpha_{1}$ proteinase inhibitor and the cell counts.

\section{Results}

The average volume of returned lavage fluid was $\mathbf{2 7 . 7}$ (SD 8.8) $\mathrm{ml}$ for the first aliquot but was greater
$(2 \mathrm{p}<0.02)$ for the second aliquot $(32.8(12.5) \mathrm{ml})$. There was no difference in recovery of fluid for either aliquot when patients with chronic bronchitis were compared with those with interstitial lung disease.

The total white cell yield did not differ between the first and the second aliquots (medians 13.0 and $12.0 \times 10^{6}$ cells; ranges $2.0-34.2$ and $3.0-37.5 \times 10^{6}$ ). The differential cell count was also similar for the two aliquots of lavage fluid (fig 1).

The protein concentrations and the $\alpha_{1}$ proteinase inhibitor $\left(\alpha_{1} \mathrm{PI}\right)$ :albumin and antileucoprotease (ALP):albumin ratios are shown in the table. There was no difference in $\alpha_{1}$ PI or albumin concentrations or in $\alpha_{1}$ PI:albumin ratio between the first and the second aliquots of lavage fluid. In contrast, however, the ALP concentration in the second aliquot was significantly lower in 15 of the patients $(2 p<0.01)$ and the ALP:albumin ratio was significantly lower in $12(2 p<0.05)$.

Thirteen of the 19 patients had red blood cells in the first aliquot, the average number of cells being $0.02(0.03) \times 10^{9} / \mathrm{ml}$. The concentrations of $\alpha_{1} \mathrm{PI}$, ALP, and albumin, as well as the $\alpha_{1}$ PI and ALP concentrations related to albumin for these samples, are shown in the table and compared with the results in the remaining six patients. The $\alpha_{1}$ PI concentration, $\alpha_{1}$ PI:albumin ratio and ALP:albumin ratio were lower in the aliquots without red blood cells $(2 p<0.05)$.

The ability of lavage fluid to inhibit porcine pancreatic elastase (PPE) was the same for the two aliquots, but showed wide variation. The median value

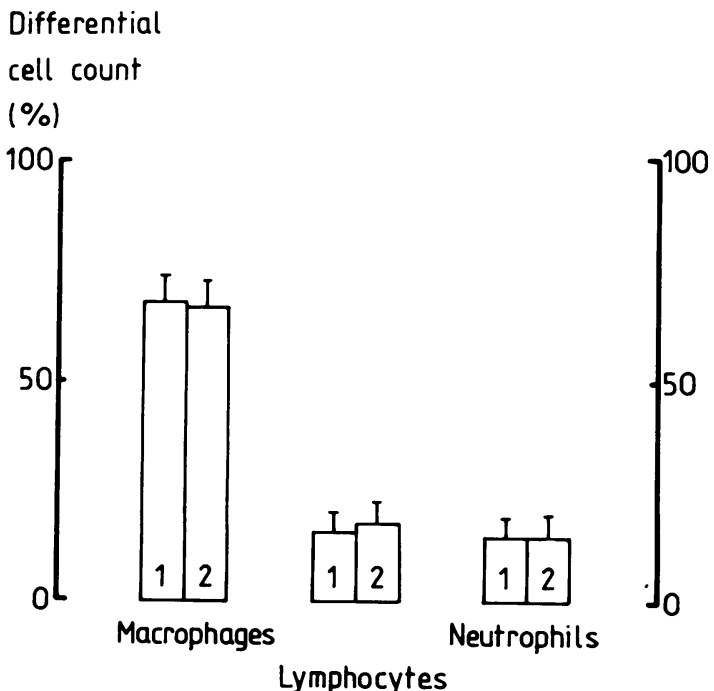

Fig 1 Differential cell counts (means and standard errors) for the first (1) and second (2) aliquots. 
Protein concentrations and ratios for the first and second aliquots and for those patients with and without red blood cells in the first aliquot (medians with ranges in parentheses)

\begin{tabular}{|c|c|c|c|c|c|}
\hline & $\begin{array}{l}\alpha_{1} P I \\
(\mu g / m l)\end{array}$ & $\begin{array}{l}A L P \\
(n g / m l)\end{array}$ & $\begin{array}{l}\text { Albumin } \\
(\mu g / m l)\end{array}$ & $\begin{array}{c}\alpha_{1} P I / A l b \\
(\mu g / m g)\end{array}$ & 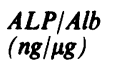 \\
\hline $\begin{array}{l}\text { 1st aliquot } \\
n=19 \\
\text { 2nd aliquot } \\
n=19\end{array}$ & $\begin{array}{l}2.4 \\
(0.1-17.9) \\
3.3 \\
(0.7-18.3)\end{array}$ & $\begin{array}{l}142.8 \\
(9.1-4625.8) \\
78.6^{*} \\
(6.4-1806.7)\end{array}$ & $\begin{array}{l}31 \\
(12-205) \\
38 \\
(14-153)\end{array}$ & $\begin{array}{l}87.3 \\
(7.1-358) \\
100.0 \\
(5.0-264)\end{array}$ & $\begin{array}{l}5.6 \\
(0.41-121.7) \\
3.4^{* *} \\
(0.14-41.1)\end{array}$ \\
\hline $\begin{array}{l}\text { RBC present } \\
n=13 \\
\text { RBC absent } \\
n=6\end{array}$ & $\begin{array}{l}2.8 \\
(0.6-17.9) \\
1.5^{* *} \\
(0.1-7.3)\end{array}$ & $\begin{array}{l}142.8 \\
(41.9-4625.8) \\
120.4 \\
(9.1-437.0)\end{array}$ & $\begin{array}{l}27 \\
(12-205) \\
42 \\
(14-191)\end{array}$ & $\begin{array}{l}125.9 \\
(21.6-358.3) \\
30.6 * * \\
(7.1-102.8)\end{array}$ & $\begin{array}{l}6.0 \\
(0.7-121.7) \\
2.37^{* *} \\
(0.4-5.8)\end{array}$ \\
\hline
\end{tabular}

$* 2 \mathrm{p}<0.01 ; * * 2 \mathrm{p}<0.05$.

RBC—red blood cells; $\alpha_{1} \mathrm{PI}-\alpha_{1}$ proteinase inhibitor; ALP-antileucoprotease; Alb-albumin.

for inhibition in the first aliquot was 0.67 (range $0-1.55) \mu \mathrm{g} P P E / \mathrm{ml}$ lavage fluid and 0.63 (range 0.06-2.80) $\mu \mathrm{g}$ PPE $/ \mathrm{ml}$ in the second aliquot. In molar terms (median values and ranges), the first aliquot inhibited $0.28(0-1.19) \mathrm{mol} \mathrm{PPE} / \mathrm{mol} \alpha_{1} \mathrm{PI}$ and the second inhibited $0.37(0.10-0.80)$ mole PPE $/ \mathrm{mol} \alpha_{1}$ PI. Much of the $\alpha_{1}$ PI present was inactive, but the proportion of inactive inhibitor was similar in the two halves of the lavage fluid (median $72.0 \%$ (range $0-100 \%)$ in the first aliquot and 63\% (18.9-89.5\%) in the second aliquot). Moreover, $\alpha_{1}$ PI function did not $\operatorname{differ}(2 p>0.1)$ between the seven bronchitic smokers and the four ex-smokers (median 0.28 (range 0.06-1.19) mol PPE inhibited $/ \mathrm{mol} \alpha_{1} \mathrm{PI}$; and 0.40 (0.16-0.51) $\mathrm{mol}$ PPE inhibited $/ \mathrm{mol} \alpha_{1}$ PI respectively).

The PPE inhibitory capacity, the total amount of $\alpha_{1} \mathrm{PI}$, and the amount of inactive $\alpha_{1} \mathrm{PI}$ were not related to the number of neutrophils or macrophages

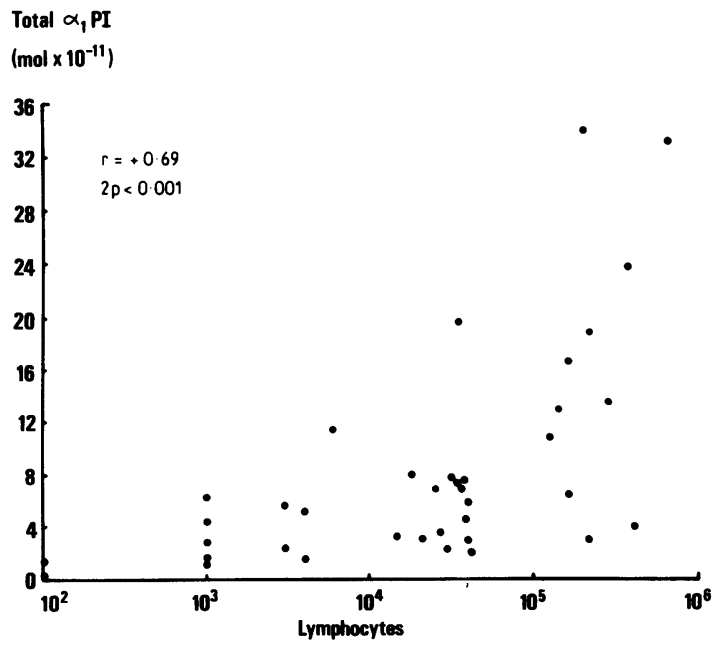

Fig 2 Relationship between total $\alpha_{1}$ proteinase inhibitor $\left(\alpha_{1} P I\right)$ and lymphocyte count per millilitre of bronchoalveolar lavage fluid for all 38 aliquots. present in the lavage fluid, but there was a significant relationship between PPE inhibitory capacity $(2 p<$ $0.01)$, total $\alpha_{1}$ PI $(2 p<0.001)$, and inactive $\alpha_{1}$ PI $(2 p$ $<0.001$ ) and the lymphocyte count. Figure 2 summarises the data for the total amount of $\alpha_{1} \mathrm{PI}$ and the lymphocyte counts in all 38 aliquots.

There was no difference in PPE inhibitory capacity (median values and ranges) in those samples containing red blood cells $(0.52(0.02-1.55) \mu \mathrm{g} \mathrm{PPE} / \mathrm{ml}$ lavage fluid and in those without red blood cells $(0.11$ $(0-2.06) \mu \mathrm{g}$ PPE/ml). In addition, the proportions of $\alpha_{1}$ PI that was inactive were similar, being $68.3 \%$ $(35.1-89.5 \%)$ and $85.0 \%(0-100 \%)$ in samples with and without red blood cells.

\section{Discussion}

Bronchoalveolar lavage is a procedure that is relatively easy to perform, and because it is well tolerated $x$ by the patient it may be used to assess disease progression and the effect of treatment by repeating lavages over a period of time. It can also be used to investigate the protein and cell content of the lower respiratory tract in normal individuals. The method 9 of collection may, however, influence the results $D$ obtained, and these must therefore be interpreted with caution. The purpose of this study was to com- No pare the first and second halves of the fluid collected during bronchoalveolar lavage-firstly, to determine o whether different aliquots of the lavage fluid come $\omega$ from different sites within the lung and, secondly to investigate whether the presence of erythrocytes (sug- 0 gesting that blood may have leaked into the airway) alters protein concentration and function.

Total and differential cell counts

The total white cell yield was similar to that reported by Davis et al for healthy smokers and non- $\stackrel{\perp}{\complement}$ smokers. ${ }^{12}$ We were unable to show a difference in total cell count between smokers and ex-smokers with 8 bronchitis, but this may merely reflect the presence of 
established airways disease in both groups. The differential cell counts remained the same throughout the lavage, confirming the results of Weinberger et al. ${ }^{13}$

\section{$\alpha_{1}$ proteinase inhibitor and albumin concentrations}

We were unable to find a difference in the concentration of either $\alpha_{1}$ proteinase inhibitor or albumin between the two halves of the lavage fluid, a result that is at variance with the findings of Davis et al ${ }^{12}$ and Merrill et al. ${ }^{5}$ The latter group showed that in normal subjects the concentrations of both total protein and albumin fell with successive aliquots but that the albumin:total protein ratio remained constant, suggesting that dilution was responsible for the observed reduction in concentration. In the present study no difference in $\alpha_{1}$ proteinase inhibitor or albumin concentration or in $\alpha_{1}$ proteinase inhibitor:albumin ratio has been shown between the aliquots, suggesting that the dilution that has occurred has been equal in the two halves of the lavage and is perhaps due to greater quantities of protein in the secretions of patients than in those of healthy subjects. Although the present study includes a mixture of patient groups, each patient acted as his or her own control. No difference was seen in the 13 patients with obstructive lung disease. However, it remains possible that differences could be found in larger groups of normal subjects or those with interstitial lung disease.

\section{Antileucoprotease concentration}

In contrast, the concentration of antileucoprotease decreased during sequential lavages in our patients. This was not due to dilution of secretion as the other protein concentrations did not alter and the antileucoprotease:albumin ratio also fell. Although it has been detected in peripheral airways by immunohistochemical techniques ${ }^{14}$ this inhibitor is thought to be located predominantly in the upper part of the respiratory tract rather than in the alveoli. ${ }^{15}$ Our results show that it is present in greater quantities relative to albumin in the first half of the lavage fluid. This might be predicted as it is thought to be associated with mucus. ${ }^{16}$ Most of our patients had cough with excess sputum production and hence greater quantities of bronchial mucus. The mucus would theoretically be harvested early in the lavage procedure and subsequent lavage would then contain relatively less mucus and more alveolar secretions, resulting in a reduction of antileucoprotease. This study provides the first evidence that secretions obtained from different regions of the lung by bronchoalveolar lavage may be biochemically different. Nevertheless, antileucoprotease could still be measured in samples recovered later from more peripheral regions of the lung, confirming the findings of previous workers in normal subjects. ${ }^{17}$

The function of $\alpha_{1}$ proteinase inhibitor and the lack of relationship to cell profile in these samples is of interest. Both macrophages and neutrophils produce oxygen radicals ${ }^{6}$ and contain enzymes capable of inactivating $\alpha_{1}$ proteinase inhibitor. ${ }^{7}$ It has been proposed that these cells may be important mediators of damage to $\alpha_{1}$ proteinase inhibitor in vivo. ${ }^{67}$ Despite this possibility, there was no relationship between these cells and the amount of inactive or total $\alpha_{1}$ proteinase inhibitor present or the PPE inhibitory capacity.

\section{Lymphocytes and $\alpha_{1}$ proteinase inhibitor}

The lymphocyte count was, however, positively correlated with the amount of inactive and total $\alpha_{1}$ PI present, and also with the PPE inhibitory capacity, expressed as $\mu \mathrm{g}$ PPE inhibited per $\mathrm{ml}$ of lavage fluid. This association was loose, although statistically significant, and its relevance therefore remains uncertain. Lymphocytes have a protease present on their cell membrane and $\alpha_{1}$ proteinase inhibitor has been shown to bind to this enzyme, modulating cell function. ${ }^{18}$ This could explain the relationship observed between total and inactive $\alpha_{1}$ PI and the number of lymphocytes present in lavage fluid. Clearly further studies will be necessary to establish the validity of the lymphocyte- $\alpha_{1}$ proteinase inhibitor relationship and, if it is confirmed, whether it represents cause and effect.

\section{$\alpha_{1}$ proteinase inhibitor function}

A large porportion of $\alpha_{1}$ proteinase inhibitor appears to be non-functional in bronchoalveolar lavage fluid from these patients (confirming the study of Boudier et $a l^{19}$ ) and the proportion of inactive inhibitor did not differ between aliquots. Carp et al reported that lavage fluid from smokers was $40 \%$ less active in its ability to inhibit enzyme than that recovered from non-smokers, ${ }^{20}$ but failed to indicate the degree of activity of $\alpha_{1}$ PI in non-smokers' lavage fluid. Those results are, however, still at variance with those of the present study, which shows that $\alpha_{1}$ PI function was no different in lavage fluid from smokers and from nonsmokers, although levels were reduced in both. Previous studies in normal subjects ${ }^{1921}$ and bronchitic patients $^{22}$ have also failed to show a difference between smokers and non-smokers. Perhaps the discrepancies are technical, relating both to the use of well characterised enzyme (as with the porcine pancreatic elastase used in the current study), and to inaccuracies in quantitation of lung $\alpha_{1}$ proteinase inhibitor. ${ }^{8}$ Without collaboration between laboratories, however, this problem may not be resolved. 


\section{Presence of erythrocytes}

The concentration of $\alpha_{1}$ proteinase inhibitor was greater in lavage samples with red blood cells present, and the $\alpha_{1}$ proteinase inhibitor:albumin and antileucoprotease:albumin ratios were also greater where red blood cells were present $(2 \mathrm{p}<0.05)$. The antileucoprotease and albumin concentrations alone were similar for the two groups. Possibly these findings relate to changes in concentration as a result of leakage of blood due to bronchoscopic trauma. Both $\alpha_{1}$ proteinase inhibitor and albumin are major components of serum but antileucoprotease is present only in small amounts in blood. ${ }^{9}$ Contamination of lavage fluid by serum should therefore theoretically increase the concentration of $\alpha_{1}$ proteinase inhibitor and albumin, reduce the antileucoprotease:albumin ratio, and have no effect on the $\alpha_{1}$ proteinase inhibitor:albumin ratio. Clearly this is not the case. The result may, however, be an artefact due to the patients studied. Most of the patients with interstitial lung disease had red blood cells in their first aliquot of lavage fluid and higher protein concentrations. When these six patients were excluded from analysis, there were eight bronchitic subjects with red blood cells in the first aliquot and five with none. Although the numbers were reduced, the only remaining difference between the groups was an increase in the antileucoprotease:albumin ratio in those aliquots with red blood cells, despite the fact that the concentrations of antileucoprotease and albumin were not significantly different. The result may be due to the cumulative effect of small changes or may be statistical artefact. Nevertheless, whether this is a real change or not, the results suggest that it would be safer to discard any samples contaminated with red blood cells.

A further technical problem is the conventional use of ultrafiltration to concentrate lavage proteins. The albumin concentrations in the samples containing red blood cells were lower than could be expected for the potential leakage of serum. This suggests a degree of protein loss and perhaps it would be preferable to study unconcentrated lavage fluid in future.

The total and differential cell counts were performed by Mrs Janet Moore in the department of pathology of the General Hospital, Birmingham. We wish to acknowledge the support of the West Midlands Regional Health Authority, the General Hospital bicentenary appeal fund, and the technical assistance of SL Hill. RAS is a Wolfson research fellow of the Royal College of Physicians of London, and HMM is a Sheldon clinical research fellow.

\section{References}

1 Hunninghake GW, Gadek JE, Kawanami O, Ferrans VJ, Crystal RG. Inflammatory and immune processes in the human lung in health and disease: evaluation by bronchoalveolar lavage. Am J Pathol 1979;97:149-205.

2 Lee CT, Fein AM, Lippmann H, Kimbel P, Weinbaum G. Elastolytic activity in pulmonary lavage fluid from patients with adult respiratory distress syndrome. $N$ Engl J Med 1981;304:192-6.

3 Stockley RA. Measurement of soluble proteins in lung secretions. Thorax 1984;39:241-7.

4 Baughman RP, Bosken CH, Loudon RG, Hurtubise P, Wesseler T. Quantitation of bronchoalveolar lavage with methylene blue. Am Rev Respir Dis 1983;128:266-70.

5 Merrill W, O'Hearn E, Rankin J, Naegel G, Matthay RA, Reynolds HY. Kinetic analysis of respiratory tract proteins recovered during a sequential lavage protocol. Am Rev Respir Dis 1982;126:617-20.

6 Carp H, Janoff A. Potential mediator of inflammation. Phagocyte-derived oxidants suppress the elastaseinhibitory capacity of alpha ${ }_{1}$-proteinase inhibitor in vitro. J Clin Invest 1980;66:987-95.

7 Banda MJ, Clark EJ, Werb Z. Limited proteolysis by macrophage elastase inactivates human $\alpha_{1}$-proteinase inhibitor. J Exp Med 1980;152:1563-70.

8 Stockley RA, Afford SC. The immunological assessment of alpha ${ }_{1}$-antitrypsin with reference to its function in bronchial secretions. Clin Sci 1983;65:373-81.

9 Kramps JA, Franken C, Dijkman JH. ELISA for quantitative measurement of low-molecular-weight bronchial protease inhibitor in human sputum. Am Rev Respir Dis 1984;129:959-63.

10 Morrison HM, Afford SC, Stockley RA. Inhibitory capacity of alpha $a_{1}$ antitrypsin in lung secretions: variability and the effect of drugs. Thorax 1984:39:510-16.

11 Hochstrasser K, Albrecht GJ, Schönberger OL, Rasche B, Lempart K. An elastase-specific inhibitor from human bronchial mucus. Isolation and characterisation. HoppeSeyler's Z Physiol Chem 1981;362:1369-75.

12 Davis GS, Giancola MS, Costanza MC, Low RB. Analyses of sequential bronchoalveolar lavage samples from healthy human volunteers. Am Rev Respir Dis 1982;126:611-6.

13 Weinberger SE, Kelman JA, Elson NA, et al. Bronchoalveolar lavage in interstitial lung disease. Ann Intern Med 1978;89:459-66.

14 Mooren HWD, Kramps JA, Franken C, Meijer CJLM, Dijkman JH. Localisation of a low-molecular-weight bronchial protease inhibitor in the human peripheral lung. Thorax 1983;38:180-3.

15 Kramps JA, Franken C, Dijkman JH. Low-molecularweight protease inhibitor in the peripheral lung [abstract]. Respiration 1984;46:S1.

16 Ohlsson K. Interactions between granulocyte proteases and protease inhibitors in the lung. Bull Eur Physiopathol Respir 1980;16, suppl:209-22.

17 Abrams WR, Fein AM, Kucich U, et al. Proteinase inhibitory function in inflammatory lung disease. IAcute bacterial pneumonia. Am Rev Respir Dis 1984; 129:735-41.

18 Bata J, Revillard J-P. Interaction between $\alpha_{1}$ antitrypsin and lymphocyte surface protease:immunoregulatory effects. Agents Actions 1981;11:614-6.

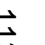

,
(1) (1) 
19 Boudier C, Pelletier A, Pauli G, Bieth JG. The functional activity of $\alpha_{1}$-proteinase inhibitor in bronchoalveolar lavage fluids from healthy human smokers and nonsmokers. Clin Chim Acta 1983;132:309-15.

20 Carp H, Miller F, Hoidal JR, Janoff A. Potential mechanism of emphysema: $\alpha_{1}$ proteinase inhibitor recovered from lungs of cigarette smokers contains oxidised methionine and has decreased elastase inhibitory capacity.
Proc Natl Acad Sci USA 1982;79:2041-5.

21 Stone PJ, Calore JD, McGowan SE, et al. Functional alpha $_{1}$-protease inhibitor in the lower respiratory tract of cigarette smokers is not decreased. Science 1983;221:1187-9.

22 Stockley RA, Afford SC. Qualitative studies of lung lavage $\alpha_{1}$-proteinase inhibitor. Hoppe-Seyler's $Z$ Physiol Chem 1984;365:503-10. 